\title{
Alterações encontradas no potencial visual evocado por padrão reverso em pacientes com esclerose múltipla definida
}

\author{
Pattern-reversal visual evoked potential abnormalities in patients \\ with defined multiple sclerosis
}

\author{
Eric Pinheiro de Andrade ${ }^{1}$ \\ Paula Yuri Sacai ${ }^{2}$ \\ Adriana Berezovsky ${ }^{3}$ \\ Solange Rios Salomão ${ }^{4}$
}

Trabalho realizado no Laboratório de Eletrofisiologia Visual Clínica do Departamento de Oftalmologia da Universidade Federal de São Paulo - UNIFESP - São Paulo (SP) - Brasil.

${ }^{1}$ Mestre, Professor Adjunto da Universidade de Santo Amaro - UNISA - São Paulo (SP) - Brasil.

2 Pós-graduanda, Tecnóloga Oftálmica do Departamento de Oftalmologia da Universidade Federal de São Paulo - UNIFESP - São Paulo (SP) - Brasil.

${ }^{3}$ Doutora, Professora Adjunta da UNIFESP - São Paulo (SP) - Brasil.

${ }^{4}$ Livre Docente, Professora Adjunta da UNIFESP - São Paulo (SP) - Brasil.

Endereço para correspondência: Eric Pinheiro de Andrade. Rua Desembargador do Vale, 900 - Apto. 42 São Paulo (SP) CEP 05010-040

E-mail: dr_eric_andrade@hotmail.com

Recebido para publicação em 06.12.2006

Última versão recebida em 27.06.2007

Aprovação em 06.08.2007

Nota Editorial: Depois de concluída a análise do artigo sob sigilo editorial e com a anuência da Dra. Maria Kiyoko Oyamada sobre a divulgação de seu nome como revisora, agradecemos sua participação neste processo.

\begin{tabular}{l} 
RESUMO \\
\hline Introdução: Esclerose múltipla é uma doença desmielinizante idiopática, \\
podendo acometer o nervo óptico com perda visual unilateral aguda que \\
pode ser observada através do potencial visual evocado (PVE), definindo \\
tal exame como de grande validade para o estudo pré-quiasmático das \\
vias visuais na esclerose múltipla. Objetivo: Analisar os achados dos \\
potenciais visuais evocados por reversão de padrões em pacientes com \\
diagnóstico prévio de esclerose múltipla e sua comparação coma acuidade \\
visual. Métodos: Vinte e quatro pacientes com diagnóstico definido de \\
esclerose múltipla foram submetidos ao PVE por reversão de padrões no \\
período de outubro de 2001 a março de 2007 . Resultados: Na resposta \\
do componente P100, nos estímulos de $15^{\prime}$ e $1^{\circ}$, se observou que 19 olhos \\
apresentaram resposta de latência até 115 ms em ambas as estimulações, \\
coincidindo também em 6 olhos que apresentaram resposta de latência \\
entre 116 a 135 ms, em 11 olhos que apresentaram resposta de latências \\
superiores a 135 ms, e em 4 olhos que não apresentaram respostas às \\
estimulações. Correlacionando a acuidade visual com a latência de P100 \\
para 15 ', obtemos na correlação de Pearson r=0,85 com p=0,000000123, \\
e para $1^{\circ}$, r=0,87 com p=0,0000000338. Conclusão: Aproximadamente \\
$60,4 \%$ dos pacientes apresentaram anormalidades no PVE por padrões \\
reversos. Contudo, a correlação entre a latência de P100 (15' e $1^{\circ}$ ) e a \\
acuidade visual foi estatisticamente significativa, assim sendo, quanto \\
melhor a acuidade visual, melhor será sua resposta aos estímulos do PVE \\
por reversão de padrões.
\end{tabular}

Descritores: Doenças desmielinizantes; Esclerose múltipla; Neurite óptica; Acuidade visual; Eletrofisiologia/métodos

\section{INTRODUÇÃ̃O}

Esclerose múltipla é uma doença desmielinizante idiopática, própria do sistema nervoso central, que altera de forma intermitente a função neurológica $^{(1-6)}$. Compromete três regiões do sistema nervoso: a) medula espinhal causa fraqueza, espasmos musculares, fadiga, distúrbios sexuais, etc; b) tronco cerebral - causa diplopia, nistagmo, ataxia, disartria e disfagia, e c) hemisférios cerebrais - causa declínio intelectual, depressão, euforia, demência, entre outras alterações. Fenômenos transitórios também podem ser referidos: epilepsia, espasmos tônicos e fenômeno de Uhthoff $f^{(1-3,7-8)}$.

Cinqüenta por cento dos pacientes com esclerose múltipla apresentam 
neurite óptica isolada, contudo outras alterações neurológicas concomitantes podem ocorrer em $75 \%$ dos pacientes do sexo feminino e em $34 \%$ do sexo masculino, classificando-os como portadores de esclerose múltipla após 15 anos de seguimento ${ }^{(1-3)}$.

O quadro clínico clássico da neurite óptica consiste na perda visual unilateral de início agudo, desconforto periocular que piora com a movimentação dos olhos, disco óptico com aspecto normal em aproximadamente $67 \%$ dos $\operatorname{casos}^{(1,3-6)}$. Dentre os exames complementares preconizados para o diagnóstico de esclerose múltipla estão a ressonância nuclear magnética, o exame do líquido cefalorraquiano por punção lombar e o potencial visual evocado ${ }^{(1-3,9-13)}$.

Em 1972, Halliday, McDonald e Mushin ${ }^{(10)}$ observando anormalidades do potencial visual evocado (PVE) devido à neurite óptica, definiram tal exame como de grande validade para o estudo pré-quiasmático das vias visuais em pacientes com esclerose múltipla ${ }^{(9,11,14)}$. O exame do potencial visual evocado por reversão de padrões, em baixa freqüência temporal de um padrão xadrez preto e branco, apresenta menor variabilidade e maior sensibilidade em detectar anormalidades na investigação clínica das vias visuais, sendo o método mais útil para avaliar a transmissão destes estímulos pela via máculo-occipital ${ }^{(5,9,15-17)}$. A principal utilidade clínica se revelou na identificação do envolvimento subclínico da via visual em pacientes suspeitos de esclerose múltipla, definindo o comprometimento multifocal do sistema nervoso central ${ }^{(5,9,18-19)}$.

O objetivo deste estudo foi analisar os achados dos potenciais visuais evocados por reversão de padrões em pacientes com diagnóstico prévio de esclerose múltipla e sua comparação com a acuidade visual.

\section{MÉTODOS}

Esta pesquisa seguiu os princípios básicos contidos na Declaração de Helsinque (1964) e foi aprovada pela Comissão de Ética em Pesquisa da UNIFESP/EPM (CEP 1649/05).

O grupo de estudo constou de 24 pacientes encaminhados ao Laboratório de Eletrofisiologia Visual Clínica do Departamento de Oftalmologia da Universidade de São Paulo - Escola Paulista de Medicina com diagnóstico definido de esclerose múltipla. Todos os pacientes foram submetidos ao exame de potencial visual evocado por reversão de padrões no período de outubro de 2001 a março de 2007. Foram 20 pacientes do sexo feminino e 4 do sexo masculino, com idades que variaram entre 16 e 60 anos (média $=40,2 \pm 10,7$ anos). $\mathrm{O}$ critério de inclusão foi ter diagnóstico definitivo de esclerose múltipla, baseado no quadro clínico, com histórico de no mínimo dois episódios de alterações neurológicas em regiões distintas do SNC e dos achados de exames de ressonância nuclear magnética de crânio e do líquido cefalorraquidiano ${ }^{(20)}$, já os critérios de exclusão englobavam: a) história pregressa de cirurgia oftalmológica e/ou opacidade de meios no olho a ser examinado; b) diagnóstico prévio de doenças sistêmicas que pudessem mascarar os resultados.
A acuidade visual foi medida em cada olho separadamente, com a melhor correção óptica, de acordo com o procedimento padrão desenvolvido pelo estudo Early Treatment Diabetic Retinopathy Study (ETDRS). A tabela de optotipos ETDRS retro-iluminada foi apresentada à distância de quatro metros.

$\mathrm{O}$ exame do potencial visual evocado por padrão reverso foi realizado em condição monocular de estimulação, num ambiente em penumbra com luminância constante em todos os testes. Cada paciente, durante o exame, permaneceu confortavelmente sentado, com o estimulador ao nível dos olhos e a um metro de distância de um monitor de vídeo de alta resolução. Foi solicitada fixação no centro da tela do monitor e durante a sessão monitorava-se constantemente a fixação e a condição de alerta.

A tela do estimulador compreendia $17^{\circ} \times 17^{\circ}$ do campo visual e o estímulo gerado compreendia padrões do tipo tabuleiro de xadrez em preto e branco com estímulos formando ângulos visuais de $15^{\prime}$ e $1^{\circ}$. Após limpeza da pele com gel abrasivo (Nuprep ${ }^{\circledR}$ ) foram fixados 3 eletrodos, de acordo com o sistema 10-20 de EEG (Jasper, 1958), sendo o primeiro no occiput médio, a dois centímetros acima do ínion $\left(\mathrm{O}_{\mathrm{z}}\right.$ - ativo), o segundo na região frontal $\left(\mathrm{FP}_{\mathrm{z}}\right.$ - referência) e o terceiro no vértex $\left(\mathrm{C}_{z}\right.$ - terra). A freqüência temporal de reversão dos padrões foi de $1,9 \mathrm{~Hz}$. Os contrastes foram utilizados ao máximo $(100 \%)$ e a iluminação foi mantida constante. As respostas occipitais foram registradas pelo sistema UTAS E3000 (LKC Technologies Inc., Gaithesburg, MD, USA). O sinal biológico foi amplificado na ordem de 40.000 vezes e filtrado com banda de passagem passa-alto em $100 \mathrm{~Hz}$ e passa-baixo em $0,3 \mathrm{~Hz}$. Foi analisada a resposta média de 100 apresentações do padrão reverso. As latências do maior componente negativo (N75) e do pico positivo (P100) foram determinadas. A amplitude foi estabelecida como a diferença do potencial entre os picos de N75 e P100.

\section{Classificação dos PVEs por reversão de padrões}

Os parâmetros avaliados a partir dos registros dos PVEs por reversão de padrões foram classificados após adaptação da classificação proposta por Tumas \& Sakamoto ${ }^{(9)}$ e alocados em três tipos:

Tipo 0: resposta normal à estimulação de $15^{\prime}$ e $1^{\circ}$.

Tipo 1: aumento na latência de P100 ao estímulo de 15, e/ou $1^{\circ}$.

Tipo 2: ausência de resposta à estimulação de $15^{\prime}$ e $1^{\circ}$.

Foram considerados como valor normal de latência do componente P100 para ambos os tamanhos de estímulo, $115 \mathrm{~ms}$, sendo que a diferença interocular de latência não pode ser superior a $3,5 \mathrm{~ms}^{(21)}$. Com relação à amplitude pico a pico, foi considerado como valor mínimo normal $3 \mu \mathrm{V}$, sendo que a diferença interocular de amplitude não pode ser superior a $30 \%$.

\section{RESULTADOS}

A tabela 1 mostra dados de idade, sexo, duração do diag- 
nóstico, acuidade visual de cada olho e presença de queixas visuais dos 24 pacientes estudados. A proporção de acometimento de mulheres/homens foi de 5:1. O tempo de diagnóstico definido de esclerose múltipla variou de 1 semana a 32 anos, com média de 9,06 $\pm 7,65$ anos e mediana de 7,5 anos. A acuidade visual variou de 20/16 até ausência de percepção luminosa. Dos 48 olhos examinados, 28 (58,33\%) apresentaram acuidade visual $20 / 20$ ou $20 / 16$. As queixas visuais estiveram presentes em $22(91,66 \%)$ pacientes, sendo a queixa visual principal mais comum $(100 \%)$ a de embaçamento, seguida de diplopia (23\%) e de outras queixas como perda de campo visual central e fotofobia (9\%).

Os dados referentes à amplitude e à latência dos componentes N75 e P100 dos 24 pacientes alocados no presente estudo estão demonstrados na tabela 2 , onde $9(37,5 \%)$ pacientes foram alocados como tipo $0,13(54,2 \%)$ como Tipo 1, e 2 $(8,3 \%)$ como tipo 2. Ao analisar a resposta do componente P100 do PVE por reversão de padrões, tanto para estímulo de $15^{\prime}$ quanto para $1^{\circ}$, se observou que 19 (39,6\%) olhos apresentaram resposta de latência do componente P100 dentro do limite normal de até $115 \mathrm{~ms}$ em ambas as estimulações. Entre latências de 116 a $135 \mathrm{~ms}$, obtivemos $6(12,5 \%)$ olhos, coincidindo novamente as duas estimulações. Tal igualdade numérica também é observada em latências superiores a $135 \mathrm{~ms}$, onde foram alocados $11(22,9 \%)$ olhos. Houve também $4(8,3 \%)$ olhos que não apresentaram respostas a ambas as estimulações.
A figura 1 mostra a distribuição de latência do componente P100 do PVE por reversão de padrões para os dois tamanhos de estímulo utilizados, agrupada por classes de até $115 \mathrm{~ms}$ (faixa normal), de 116 a $135 \mathrm{~ms}$ (prolongamento moderado da velocidade de condução), acima de $135 \mathrm{~ms}$ (prolongamento grave da velocidade de condução) e não detectável.

O teste de correlação de Pearson foi utilizado para análise dos valores de latência do componente P100 do PVE de reversão de padrões com a acuidade visual $\log$ MAR do olho com melhor acuidade. Para os dois pacientes que tiveram respostas não detectáveis no PVE foi estabelecido o valor arbitrário de $300 \mathrm{~ms}$ para o componente $\mathrm{P} 100$ e de $3 \log \mathrm{MAR}$ para a acuidade visual. $\mathrm{O}$ valor de $\mathrm{r}=0,85(\mathrm{p}=0,000000123)$ foi encontrado para os estímulos de $15^{\prime}$ de ângulo visual e $\mathrm{r}=0,87(\mathrm{p}=0,0000000338)$ para os estímulos de $1^{\circ}$ de ângulo visual. A figura 2 mostra a distribuição de latência de P100 e da acuidade visual dos 22 pacientes que tiveram respostas detectáveis no PVE por reversão de padrões para os dois estímulos empregados.

A diferença interocular de latência foi calculada para os dois tamanhos de estímulos, havendo diferenças maiores do que $3,5 \mathrm{~ms}$ em $11(50 \%)$ dos 22 pacientes para ambos os estímulos. A assimetria maior do que $30 \%$ na amplitude pico a pico do PVE por reversão de padrões foi constatada em 6 $(27,3 \%)$ pacientes para o estímulo de ângulo visual de $15^{\prime}$ e em $4(18,2 \%)$ pacientes para o estímulo de ângulo visual de $1^{\circ}$.

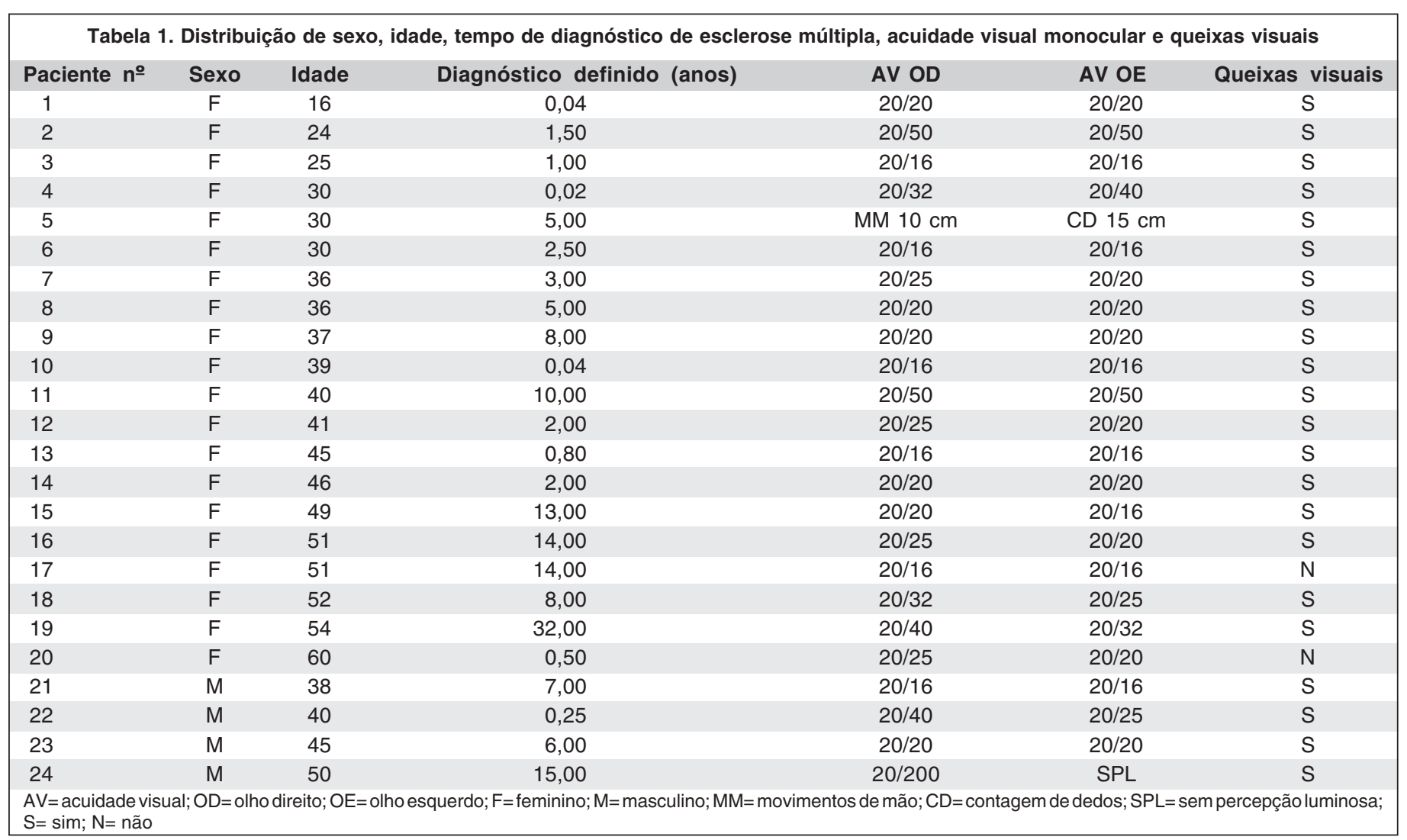




\begin{tabular}{|c|c|c|c|c|c|c|c|c|c|c|c|c|}
\hline \multirow[b]{2}{*}{ Pac. $\mathrm{n}^{\circ}$} & N75 & P100 & N75-P100 & N75 & P100 & N75-P100 & N75 & P100 & N75-P100 & N75 & P100 & N75-P100 \\
\hline & $\begin{array}{l}\text { Latência } \\
\text { ms }\end{array}$ & $\begin{array}{l}\text { Latência } \\
\text { ms }\end{array}$ & $\begin{array}{l}\text { Amplitude } \\
\qquad \mathrm{V}\end{array}$ & $\begin{array}{l}\text { Latência } \\
\text { ms }\end{array}$ & $\begin{array}{l}\text { Latência } \\
\text { ms }\end{array}$ & $\begin{array}{l}\text { Amplitude } \\
\qquad \mu \mathrm{V}\end{array}$ & $\begin{array}{l}\text { Latência } \\
\text { ms }\end{array}$ & \multicolumn{2}{|c|}{$\begin{array}{c}\text { Latência Amplitude } \\
\text { ms } \mu \mathrm{V}\end{array}$} & $\begin{array}{l}\text { Latência } \\
\text { ms }\end{array}$ & $\begin{array}{l}\text { Latência } \\
\text { ms }\end{array}$ & $\begin{array}{l}\text { Amplitude } \\
\qquad \mu \mathrm{V}\end{array}$ \\
\hline \multicolumn{13}{|c|}{ Tipo 0 - respostas normais à estimulação de $15^{\prime}$ e de $1^{\circ}$} \\
\hline 8 & 76,0 & 103,5 & 7,1 & 61,0 & 106,0 & 7,6 & 76,5 & 104,5 & 6,2 & 68,5 & 111,0 & 6,4 \\
\hline 10 & 79,5 & 102,0 & 19,0 & 73,5 & 105,0 & 13,9 & 77,5 & 106,5 & 11,1 & 74,0 & 107,0 & 10,8 \\
\hline 14 & 86,0 & 111,0 & 10,8 & 78,5 & 106,0 & 10,1 & 87,5 & 107,0 & 11,7 & 81,5 & 102,0 & 7,6 \\
\hline 18 & 92,0 & 114,0 & 7,0 & 82,0 & 109,0 & 9,5 & 74,0 & 113,0 & 7,4 & 84,0 & 112,0 & 7,9 \\
\hline 21 & 70,0 & 114,0 & 11,5 & 76,5 & 112,0 & 10,1 & 60,0 & 114,0 & 12,9 & 72,0 & 110,0 & 9,2 \\
\hline 22 & 79,5 & 101,5 & 10,0 & 78,5 & 99,5 & 9,8 & 86,0 & 109,5 & 7,6 & 78,5 & 107,0 & 13,3 \\
\hline 3 & 81,5 & 148,0 & 7,7 & 71,0 & 129,0 & 5,8 & 76,5 & 147,5 & 9,0 & 82,0 & 132,5 & 8,7 \\
\hline 4 & 88,5 & 113,5 & 7,2 & 84,5 & 107,0 & 7,3 & 89,0 & 119,5 & 5,0 & 85,5 & 120,0 & 6,5 \\
\hline 7 & 115,5 & 196,5 & 3,1 & 108,5 & 138,0 & 3,8 & 106,5 & 163,0 & 5,4 & 124,0 & 164,5 & 4,5 \\
\hline 9 & 94,5 & 139,5 & 6,5 & 92,0 & 164,0 & 5,3 & 112,0 & 143,0 & 11,7 & 91,0 & 162,0 & 4,9 \\
\hline 11 & 103,0 & 147,0 & 5,9 & 76,5 & 164,5 & 7,1 & 116,0 & 168,5 & 3,1 & 75,0 & 187,0 & 9,8 \\
\hline 12 & 144,0 & 171,0 & 4,0 & 110,5 & 162,0 & 3,0 & 99,0 & 130,5 & 4,5 & 90,5 & 127,0 & 3,6 \\
\hline 13 & 84,5 & 118,5 & 8,7 & 76,5 & 127,0 & 8,8 & 84,5 & 110,0 & 12,0 & 69,5 & 116,5 & 10,3 \\
\hline 15 & 87,5 & 134,5 & 3,2 & 115,5 & 142,0 & 3,2 & 83,0 & 134,0 & 4,5 & 99,0 & 138,5 & 5,0 \\
\hline 16 & 124,5 & 166,5 & 3,3 & 87,0 & 107,5 & 3,6 & 95,0 & 131,5 & 4,3 & 76,0 & 105,5 & 7,6 \\
\hline 17 & 91,5 & 117,0 & 25,5 & 85,0 & 124,0 & 17,9 & 90,0 & 118,0 & 18,5 & 82,0 & 125,0 & 15,3 \\
\hline 19 & 125,0 & 167,0 & 5,4 & 116,0 & 146,0 & 7,4 & 127,5 & 166,5 & 11,1 & 116,0 & 146,0 & 12,2 \\
\hline 20 & 92,0 & 121,5 & 20,6 & 66,5 & 119,0 & 17,7 & 87,5 & 119,0 & 22,3 & 61,0 & 114,0 & 17,4 \\
\hline \multicolumn{13}{|c|}{ Tipo 2 - Ausência de resposta } \\
\hline
\end{tabular}

\section{DISCUSSÃO}

O presente estudo analisou 24 pacientes com quadro clínico definido de esclerose múltipla, sendo 20 do sexo feminino $(83 \%)$ e 4 do sexo masculino (17\%), confirmando relatos anteriores de maior incidência da doença em mulheres ${ }^{(1-3)}$.

Dezenove $(39,6 \%)$ olhos apresentaram resultados normais no potencial visual evocado por padrão reverso para ambos os estímulos, enquanto que em $21(43,8 \%)$ o exame detectou anormalidades em ambas as estimulações, e em 8 $(16,6 \%)$ olhos houve anormalidades na estimulação de $15^{\prime}$ ou $1^{\circ}$, totalizando $29(60,4 \%)$ olhos que apresentaram alguma alteração no potencial visual evocado por padrão reverso, o que contrasta com outro estudo que evidenciou alterações em $96,4 \%$ dos pacientes ${ }^{(9)}$. Contudo, a literatura afirma que a freqüência de anormalidades do potencial visual evocado por padrão reverso em pacientes com esclerose múltipla definida varia entre $57 \%$ e $100 \%{ }^{(22)}$, sendo essa variabilidade explicada devido à diversidade metodológica da realização dos testes e principalmente da seleção das amostras ${ }^{(22)}$.
Contudo, o presente estudo apresentou uma correlação de Pearson entre a latência de P100 (para ambas as estimulações) e a acuidade visual (em logMar) estatisticamente significativa, assim sendo, quanto melhor a acuidade visual apresentada pelo paciente, melhor será a nitidez da imagem observada e melhor será sua resposta aos estímulos do PVE por reversão de padrões, influência também relatada em outro estudo ${ }^{(23)}$.

Com relação à importância do exame de potencial visual evocado por padrão reverso em pacientes com suspeita de esclerose múltipla, notamos através deste estudo realizado em pacientes com o diagnóstico estabelecido, um método relativamente eficaz, apresentando positividade em $60,4 \%$ dos pacientes estudados. Contudo, a acuidade visual desempenha um importante fator na realização do exame, visto que permite ao paciente obter uma imagem mais nítida do estímulo, pois o borramento visual pode influenciar negativamente nos valores dos parâmetros de amplitude e latência.

O potencial visual evocado por reversão de padrões é um exame que está consagrado na literatura como coadjuvante do diagnóstico de esclerose múltipla, juntamente com a análise 


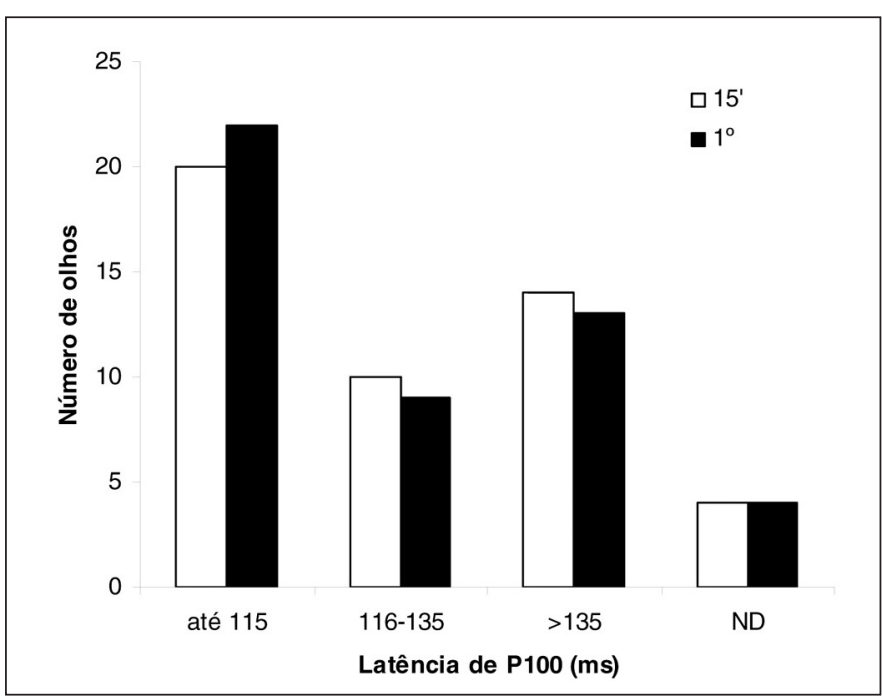

Figura 1 - Distribuição da latência (ms) do componente P100 do PVE por reversão de padrões para estímulos de ângulo visual de $15^{\prime}$ e de $1^{\circ}$ de olhos de 24 pacientes com diagnóstico definido de esclerose múltipla

do líquido cefalorraquidiano e a imagem de ressonância nuclear magnética de crânio, tendo um papel superior ao da neuroimagem, quando este último era realizado de forma rudimentar. Atualmente, devido ao grande progresso das tecnologias do exame de ressonância nuclear magnética, esta se tornou o principal arsenal de diagnose, contudo, em alguns casos, principalmente naqueles onde a imagem não é conclusiva, devemos ressaltar o uso do PVE, em concomitância com a clínica apresentada pelo paciente, ao exame do líquido cefalorraquidiano, por não ser invasivo e doloroso.

\section{ABSTRACT}

Introduction: Multiple sclerosis is an idiopathic demyelinating disease that may affect the optic nerve leading to acute unilateral visual loss, which could be observed by means of evoked visual potential (VEP). This exam is much valued for studying prechiasmatic visual paths in multiple sclerosis. Purpose: To analyze the findings of pattern reversal VEP in patients with prior diagnosis of multiple sclerosis and to compare them to visual acuity. Methods: Twentyfour patients with a definite diagnosis of multiple sclerosis were submitted to pattern reversal VEP from October 2001 to March 2007. Results: In P100 component response, at 15' and $1^{\circ}$ stimuli, 19 eyes presented latency response up to 115 $\mathrm{ms}$ in both stimuli, which coincided in 6 eyes with latency response between 116 and $135 \mathrm{~ms} ; 11$ eyes had a latency response higher than $135 \mathrm{~ms}$, and four eyes did not respond to stimuli. Correlating visual acuity with P100 latency for $15^{\prime}$, in Pearson $\mathrm{r}$ correlation, $\mathrm{r}=0.85$ with $\mathrm{p}=0.000000123$, and for $1^{\circ}, r=0.87$ with $\mathrm{p}=0.0000000338$. Conclusion: Approximately $60.4 \%$ of patients presented abnormalities. However, the correlation between the P100 latency $\left(15^{\prime}\right.$ and $\left.1^{\circ}\right)$ and

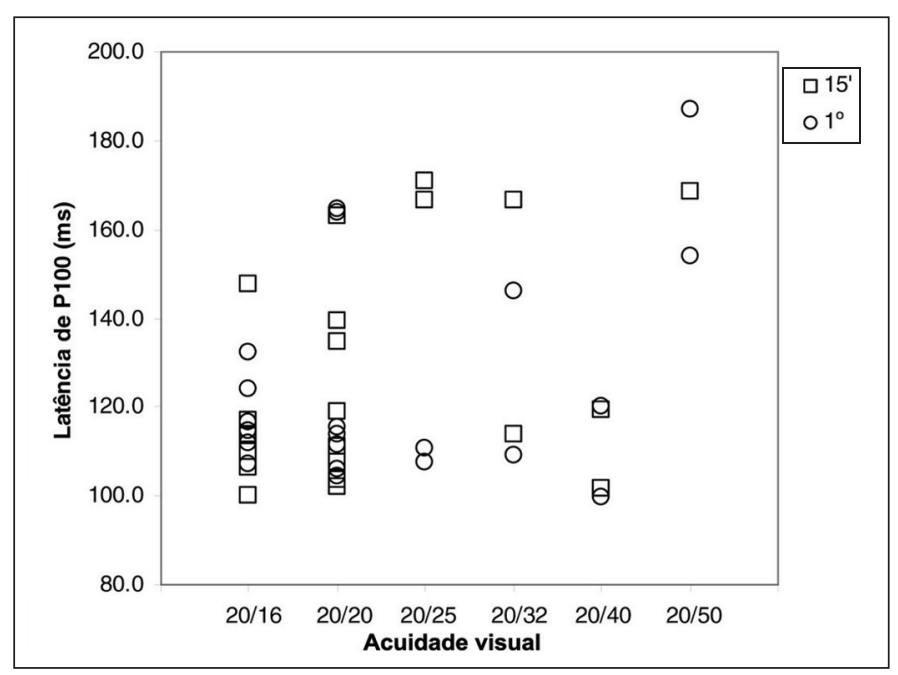

Figura 2 - Distribuição dos valores de acuidade visual (equivalente de Snellen da tabela logMAR) e da latência do componente P100 (ms) do PVE de reversão de padrões do olho de melhor acuidade visual de 22 pacientes com diagnóstico definido de esclerose múltipla

visual acuity was statistically significant; therefore the better the visual acuity, the better the response to stimuli of the pattern-reversal VEP.

Keywords: Demyelinating diseases; Multiple sclerosis; Optic neuritis; Visual acuity; Electrophysiology/methods

\section{REFERÊNCIAS}

1. Miller NR, Newman NJ. The essentials: Wash and Hoyt's clinical neuroophthalmology. $5^{\text {th }}$ ed. Baltimore: Williams \& Wilkins; 1998. p.198.

2. Dantas AM, Spielmann A, Lalanne M, Corbe C. Neuro-oftalmologia. Rio de Janeiro: Cultura Medica; 1999. p.316.

3. Dantas AM, Zangalli AL, editores. Neuro-oftalmologia. Rio de Janeiro: Cultura Médica; 1999. v.1, p.65.

4. Beck RW. The optic neuritis treatment trial. Arch Ophthalmol. 1988;106(8): 1051-3.

5. Fuhr P, Borggrefe-Chappuis A, Schindler C, Kappos L. Visual and motor evoked potentials in the course of multiple sclerosis. Brain. 2001;124(Pt 11):2162-8.

6. Brusa A, Jones SJ, Plant GT. Long-term remyelination after optic neuritis: A 2-year visual evoked potential and psychophysical serial study. Brain. 2001; 124(Pt 3):468-79.

7. Dujmovic I, Mesaros S, Pekmesovic T, Levic Z, Drulovic J. Primary progressive multiple sclerosis: clinical and paraclinical characteristics with application of the new diagnostic criteria. Eur J Neurol. 2004;11(7):439-44.

8. Rousseff RT, Tzvetanov P, Rousseva MA. The bifid visual evoked potential - normal variant or a sign of demyelination? Clin Neurol Neurosurg. 2005; 107(2):113-6.

9. Tumas V, Sakamoto AC. Anormalidades do potencial visual por padrão reverso em pacientes com esclerose múltipla definida. Arq Neuropsiquiatr. 1995; 53(4):743-8.

10. Halliday AM, McDonald WI, Mushin J. Delayed visual evoked response in optic neuritis. Lancet. 1972;1(7758):982-5.

11. Cuypers MH, Dickson K, Pinckers AJ, Thijssen JM, Hommes OR. Discriminative power of visual evoked potential characteristics in multiple sclerosis. Doc Ophthalmol. 1995;90(3):247-57.

12. Asselman P, Chadwick DW, Marsden CD. Visual evoked responses in the diagnosis and management of patients suspected of multiple sclerosis. Brain. 1975;98(2):261-82.

13. Duwaer AL, Spekreijse H. Latency of luminance and contrast evoked potentials in multiple sclerosis patients. Electroencephalogr Clin Neurophysiol. 1978;45(2):244-58. 
948 Alterações encontradas no potencial visual evocado por padrão reverso em pacientes com esclerose múltipla definida

14. Leys MJ, Candaele CM, De Rouck AF, Odom JV. Detection of hidden visual loss in multiple sclerosis. A comparison of pattern-reversal visual evoked potentials and contrast sensitivity. Doc Ophthalmol. 1991;77(3):255-64.

15. Hume AL, Waxman SG. Evoked potentials in suspected multiple sclerosis: diagnostic value and prediction of clinical course. J Neurol Sci. 1988;83(2-3): 191-210.

16. Wilson WB, Keyser RB. Comparison of the pattern and diffuse-light visual evoked responses in definite multiple sclerosis. Arch Neurol. 1980;37(1):30-4.

17. Chiappa KH. Pattern-shift visual evoked potentials: methodology. In: Chiappa $\mathrm{KH}$, editor. Evoked potentials in clinical medicine. $2^{\text {nd }}$ ed. New York: Raven Press; 1990. p.37-109.

18. Aminoff MJ. Evoked potential studies in neurological diagnosis and management. Ann Neurol. 1990;28(5):706-10.

19. Novak GP, Wiznitzer M, Kurtzberg D, Giesser BS, Vaughan HG Jr. The utility of visual evoked potentials using hemifield stimulation and several check sizes in the evaluation of suspected multiple sclerosis. Electroencephalogr Clin Neurophysiol. 1998;71(1):1-9.

20. Associação Brasileira de Esclerose Múltipla (ABEM). Diagnóstico de esclerose múltipla[texto na Internet]. [citado 2006 Jun 12]. Disponível em: http:// www.abem.org.br/versao/pt/esclerose/diagnostico.asp

21. Salomão SR, Sacai PY, Pereira JM, Berezovsky A. Pattern-reversal visually evoked potentials in healthy adults [abstract]. Invest Ophthalmol Vis Sci. $2006 ; 47$.

22. Iragui-Madoz VJ. Electrophysiology of multiple sclerosis. In: Daly DD, Pedley TA, editors. Current practice of clinical electroencephalography. $2^{\text {nd }} \mathrm{ed}$ New York: Raven Press; 1990. p.708-38.

23. Luccas FJC, Rodrigues Alves CA. Potencial evocado visual por padrão reverso (PEV-PR): influência da acuidade visual. Rev Bras Oftalmol. 1986;45(3):97-103. 